\title{
Implementation of MIN/MAX functions using Operational Transconductance Amplifier
}

\author{
Shruti Jain \\ Jaypee University of Information Technology, Solan, Himachal Pradesh, India
}

\begin{abstract}
This paper shows the different steps to realize and simulate the MIN/MAX fuzzy operators using operational transconductance amplifier, operational amplifier and 2 stage CMOS operational amplifier using SPICE. This paper also shows the calculation of different parameters like slew rate, power dissi, CMRR and gain for all the fuzzy operators. Later comparison of all parameters was discussed. The results with OTA comes out to be the best as it uses less voltage, more slew rate for high speed operations.
\end{abstract}

Keywords: Operational transconductance amplifier, CMOS operational amplifier, MIN, MAX.

\section{INTRODUCTION}

An operational amplifier (op-amp) is a directly coupled For an ideal VCCS, $R_{i}$ and $R_{o}$ should be infinite so that $G_{m}$ amplifier which includes different blocks like : dual input approaches $G_{M}$. The architecture which will implement the balanced output, dual input unbalanced output, level OTA should have high input impedance, high translator and a push pull amplifier. An op-amp can transconductance gain, and high output impedance. Fig 2 amplify both AC and DC and helps in performing all $(b)$ illustrates the OTA architecture.

mathematical operations. [1, 2, 3].

The two stage operational amplifier $[4,5]$ consists of four different parts as shown in Fig 1 :

1. Differential amp which converts voltage to current

2. Current mirror circuit which converts current to voltage.

3. Transconductance amplifier with gate grounded which converts voltage to current.

4. Class A amplifier with source or sink load which converts current to voltage.

Operational Transconductance Amplifier (OTA) is also called as voltage controlled current source (VCCS) $[3,6$, 7, 8]. Fig $2(a)$ shows the use of a OTA which is represented by the circuit within the shaded box. $R_{i}$ is the input resistance, $G_{\mathrm{m}}$ is the gain, and $R_{o}$ is the output resistance. $R_{S}$ represents the resistance of the external source $V_{S}$ and $R_{L}$ represents the load resistance. The VCCS gain can be expressed as

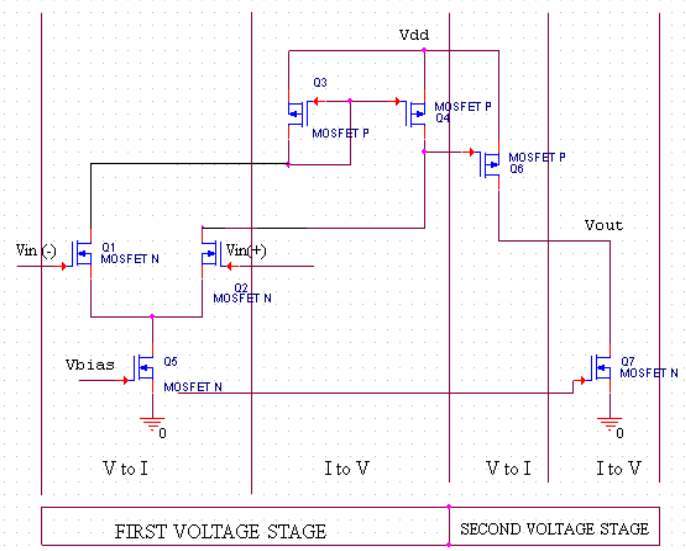

Fig 1 : Two stage CMOS operational amplifier

$$
G_{M}=\frac{G_{m} R_{o} R_{i}}{\left(R_{i}+R_{S}\right)\left(R_{o}+R_{L}\right)}
$$

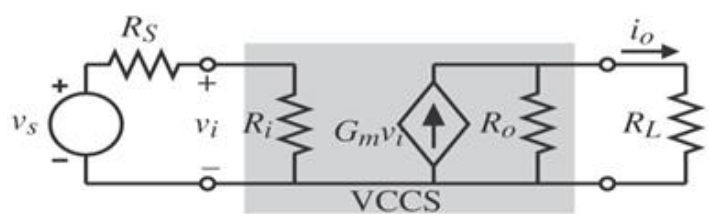

(a)

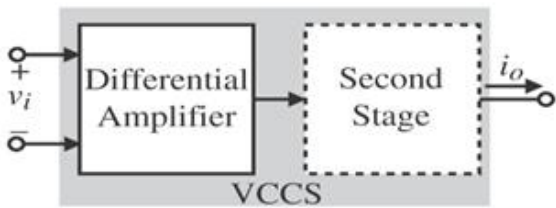

(b)

Fig 2 VCCS (a) Circuit (b) Possible architecture

The input stage is differential amplifier with current mirror load. If we need large gain, then second stage was used as inverter otherwise not required. But if we need large gain and high output impedance than cascade load was used as second stage. The block diagram is shown in Fig 2(b).

The fuzzy set is represented as $[9,10]$

$$
A=\left\{x, \mu_{A}(x)\right\} \quad x \in X
$$

where $x$ is an element in $X$ and $\mu_{\mathrm{A}}(x)$ is the membership function of set. The fuzzy set using discrete membership function is expressed as :

$$
A=\sum_{x_{i} \in X} \mu_{A}(x) / x_{i}
$$

The fuzzy set using continuous membership function is expressed as :

$$
A=\int \mu_{A}(x) / x_{i}
$$

Fuzzy system is divided into five parts as shown in Fig 3. 
INTERNATIONAL JOURNAL OF INNOVATIVE RESEARCH IN ELECTRICAL, ELECTRONICS, INSTRUMENTATION AND CONTROL ENGINEERING Vol. 4, Issue 1, January 2016

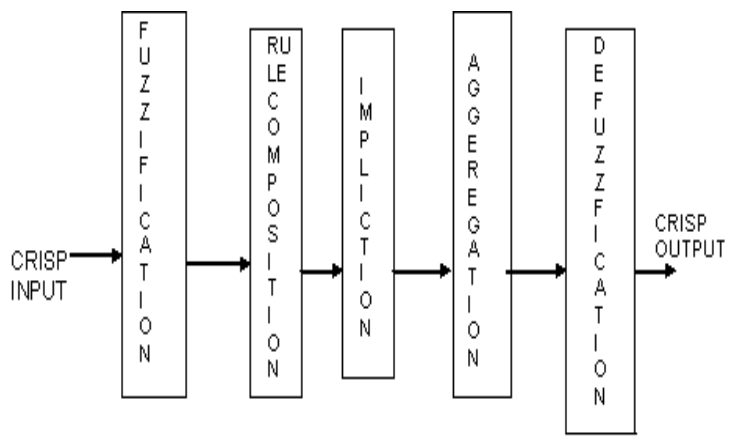

Fig 3 Fuzzy System

This paper discusses the various steps for realizing MIN/ MAX fuzzy operator, implementing it using different methods like OTA, 2 stage CMOS op-amp and op-amp using BJT and finally simulating it with PSPICE software. PSPICE is used to calculate voltage and current waveform using different analysis like Transient Analysis, AC Analysis, DC analysis etc [11].

\section{MATERIAL AND METHODOLOGY}

This section deals with the analysis and design of the MAX \& MIN circuits. The electronic implementation of these circuits is essential as these are the building blocks of FLC.

1 ) MAX circuit : A two input MAX ckt can be realized by using the OR circuit output to the non-inverting terminal of an op-amp.

Let $V_{1}$ and $V_{2}$ the two voltages to the diodes $D_{1} \& D_{2}$ respectively. If $V_{1}>V_{2}$, then the diode $\mathrm{D}_{1}$ will be more forward biased and voltage $V_{\mathrm{A}}=V_{1}-V_{\gamma}$ will appear at node $A$, where $V_{\gamma}$ is the biased voltage of diode $D_{1}$.

In order that the voltage input at node $B, V_{\mathrm{B}}$, to the non inverting terminal is equal to the $V_{1}$, a diode $D_{3}$, similar to diodes $D_{1}$ and $D_{2}$ is connected in parallel, across node $A$, so that the forward bias voltage (cut-in voltage, $V_{\gamma}$ ) developed across $\mathrm{D}_{3}$ is added at node $B$ to the output from the diode logic OR gate.

As a result, the op-amp effectively behaves as an adder circuit, with a gain of 1 , in which the voltages $V_{\mathrm{A}}$ and $V_{\gamma}$ are added and the output $V_{1}$ is obtained, which is the maximum of the two voltages $V_{1}$ and $V_{2}$.

For $V_{1}>V_{2}$

Voltage at node $A, V_{\mathrm{A}}=V_{1}-V_{\gamma}$

Voltage developed across diode $\mathrm{D}_{3}$ at node $C, V_{\mathrm{C}}=V_{\gamma}$

Using the superposition theorem at node $\mathrm{B}$, The voltage at node $B$, due to $V_{\mathrm{A}}$, when $V_{\mathrm{C}}=0$ is given by

$$
V_{\mathrm{BA}}=\left(V_{\mathrm{A}} / R+R\right)^{*} R=V_{\mathrm{A}} / 2
$$

The voltage at node $B$, due to $V_{\mathrm{C}}$, when $V_{\mathrm{A}}=0$ is given by

$$
V_{\mathrm{BC}}=\left(V_{\mathrm{C}} / R+R\right)^{*} R=V_{\mathrm{C}} / 2
$$

Therefore,

$$
V_{\mathrm{B}}=V_{\mathrm{BA}}+V_{\mathrm{BC}}=\left(V_{\mathrm{A}}+V_{\mathrm{C}}\right) / 2
$$

Now, the output of the op-amp is given by

$$
V_{\mathrm{O}}=\left(1+R_{\mathrm{F}} / R_{1}\right) *\left(V_{\mathrm{A}}+V_{\mathrm{C}}\right) / 2
$$

where $\left(1+R_{F} / R_{1}\right)$ is the gain of the non-inverting amplifier

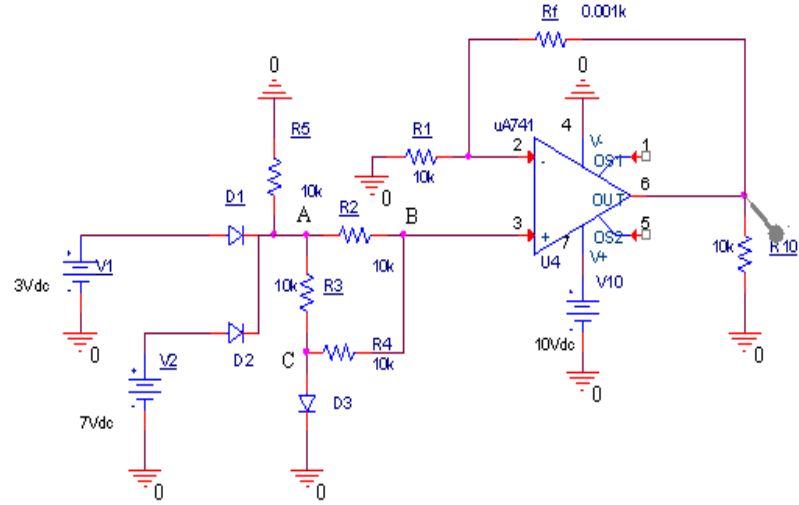

Fig 4 Circuit Diagram for MAX operator using op-amp

For $R_{\mathrm{F}}=R_{1}$

$$
\begin{gathered}
V_{\mathrm{O}}=2 *\left(V_{\mathrm{A}}+V_{\mathrm{C}}\right) / 2 \\
V_{\mathrm{O}}=V_{\mathrm{A}}+V_{\mathrm{C}} \\
V_{\mathrm{O}}=V_{1}
\end{gathered}
$$

Thus, the output $V_{\mathrm{O}}$ of the op-amp is equal to the MAX of the two voltages $V_{1}$ and $V_{2}$.

Now let us assume that $V_{2}=7$ volts and $V_{1}=3$ volts, then the circuit shown in Fig 4 will give us

$V_{\mathrm{O}}=\operatorname{Max}(7,3)=7$ volts

Its corresponding output illustrates in Fig 5

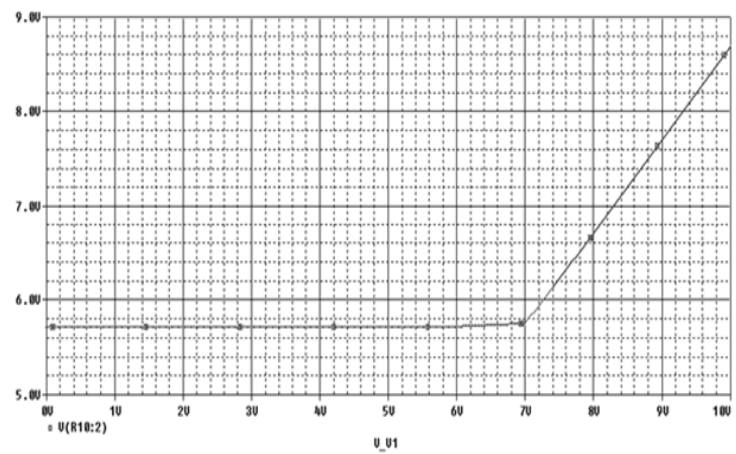

Fig 5 Output corresponding to MAX operator using op-amp

Similarly, we have designed MAX function using 2 stage op-amp (result shown in fig 6) and OTA (result shown in fig 7) and also calculated its electrical parameters shown in fig 8 . Fig 8 illustrates that OTA is the best, as slew rate is more which proves speed is more; CMRR is more which proves that common mode voltage can be easily removed.

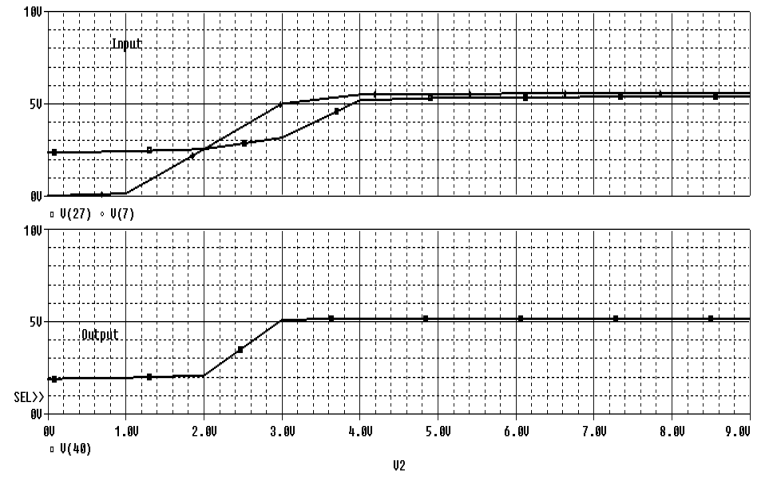

Fig 6 Output corresponding to MAX function using 2 stage CMOS op-amp 
INTERNATIONAL JOURNAL OF INNOVATIVE RESEARCH IN ELECTRICAL, ELECTRONICS, INSTRUMENTATION AND CONTROL ENGINEERING Vol. 4, Issue 1, January 2016

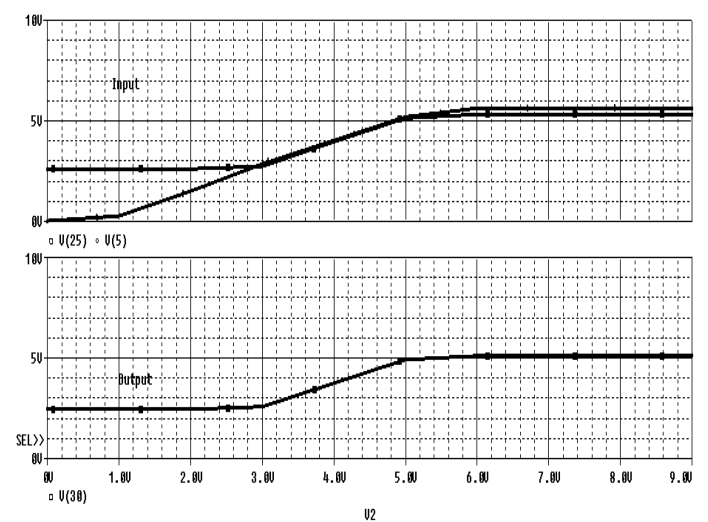

Fig 7 MAX function using OTA

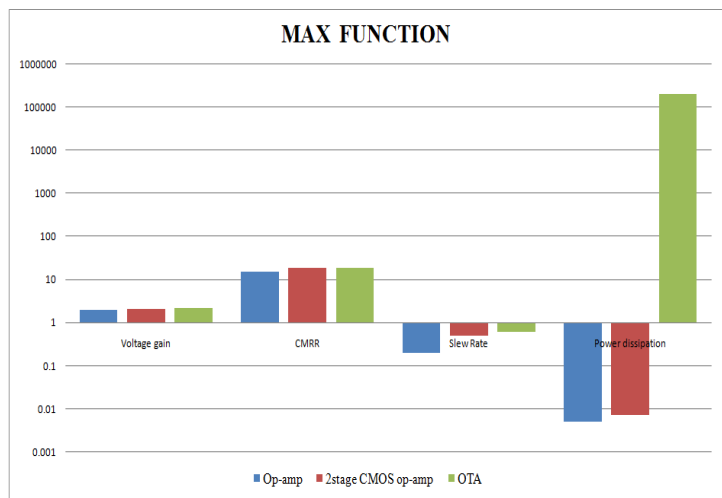

Fig 8 Comparison of MAX operator in terms of electrical parameters

2) MIN circuit : The MIN circuit can be realized by using diode in AND logic configuration which was given to the non-inverting terminal of an op-amp.

Let $V_{1} \& V_{2}$ be the two voltage inputs to the diodes $D_{1} \&$ $D_{2}$ resp. The voltage at node A is the MIN of two inputs voltages $V_{1} \& V_{2}$ (AND logic). If $V_{1}<V_{2}$, then $V_{1}$ will appear at output i.e. node A otherwise $V_{2}$ will appear. The actual voltage at node $\mathrm{A}$ is $V_{1}+V_{\gamma}$ where $V_{\gamma}$ is the biased voltage of the diode. $V_{\mathrm{A}}$ is the input to the non-inverting terminal of the op-map. The input at non- inverting terminal should be equal to output so we have assumed the gain as 1 i.e. the output of op-amp at node B is $V_{1}+V_{\gamma}$. To get output as $V_{1}$, diode $D_{3}$ is a attached at the output with resistance $R_{\mathrm{L}}$ in series with that. The final output is taken across load $R_{\mathrm{L}}$ so that

$$
V_{\mathrm{O}}=V_{\mathrm{O}}-V_{\mathrm{D} 3}=V_{\mathrm{A}^{-}}-V_{\gamma}=V_{1}+V_{\gamma}-V_{\gamma}=V_{\mathrm{O}} .
$$

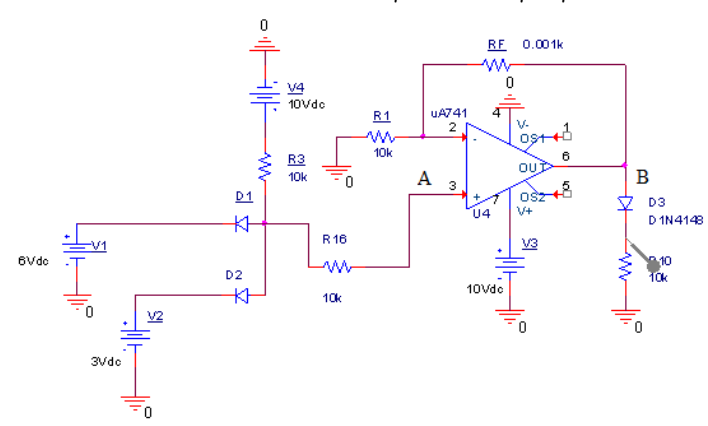

Fig 9 Circuit diagram of MIN using op-amp

Let us assume $V_{2}=3$ volts and $V_{1}=6$ volts,

then output

$V_{\mathrm{O}}=\operatorname{MIN}(6,3)=3$ volts shown in Fig 10.

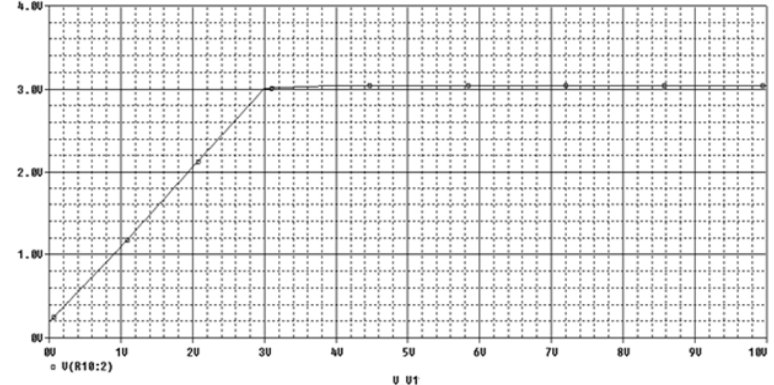

Fig 10 : Output of MIN circuit using op-amp

Similarly, we have designed MIN function using 2 stage op-amp (result shown in fig 11) and OTA (result shown in fig 12) and also calculated its electrical parameters shown in fig 13. Fig 13 illustrates that OTA is the best as slew rate is more which proves speed is more; CMRR is more which proves that common mode voltage can be easily removed.

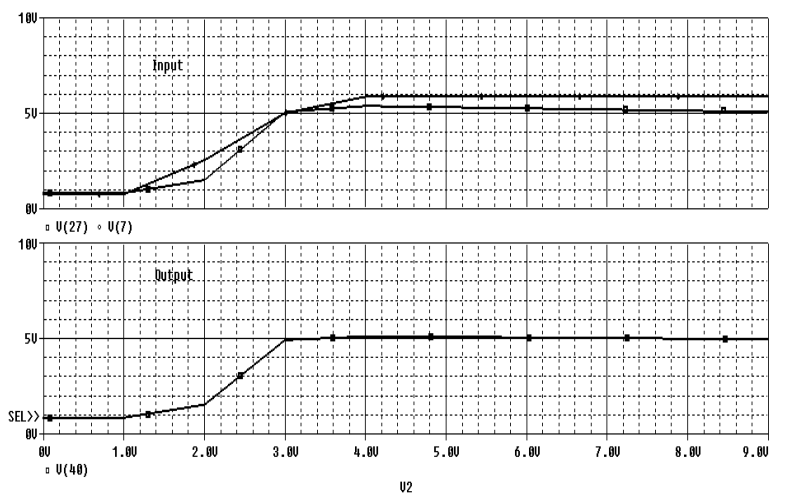

Fig 11 MIN function using 2 stage op-amp

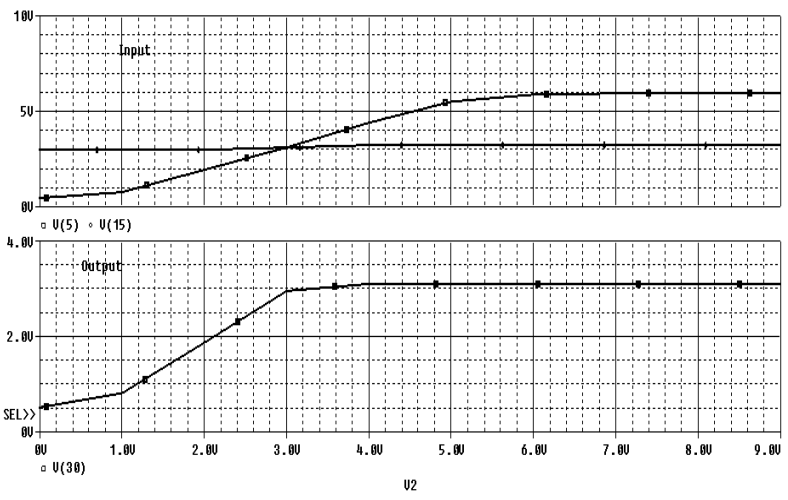

Fig 12 MIN function using OTA

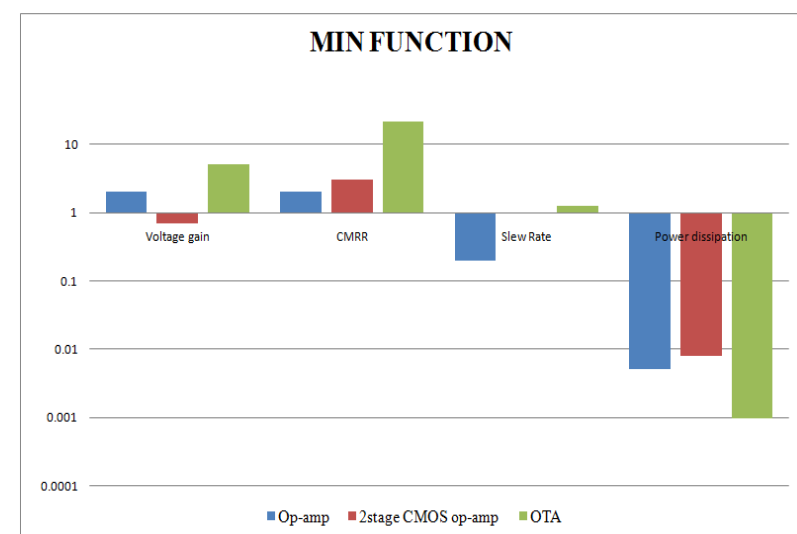

Fig 13 Comparison table of MIN operator in terms of electrical parameters 


\section{CONCLUSION AND FUTURE SCOPE}

In this paper we have presented the electronic circuit for MIN/MAX operator using op-amp, OTA and two stage CMOS op-amp. We have also implemented the MIN/ MAX functions and compare the results with two stage CMOS op-amp and op-amp using BJT. Results with OTA come out to be the best in all aspect.

\section{REFERENCES}

[1] Gayakwad, R.A., Op-Amps and Linear Integrated Circuits, 2002. 3rd ed., Prentice Hall of India Pvt. Ltd, New Delhi.

[2] Sedra S Smith, Microelectronic circuits, 2004. 5th ed. UK: Oxford University Press.

[3] Jain S, "Design and simulation of fuzzy membership functions for the fuzzification module of fuzzy system using operational amplifier", International Journal of Systems, Control and Communications (IJSCC), 69-83, 6(1):2014.

[4] Allen P.E, holberg D.R., CMOS analog circuit design , 2011. International Student edition, Oxford.

[5] Jain S, "Design and Simulation of Fuzzy Implication Function of Fuzzy System Using Two Stage CMOS Operational Amplifier", International Journal of Emerging Technologies in Computational and Applied Sciences (IJETCAS), 150-155, 7(2): Dec 2013- Feb 2014.

[6] Jain S, Chauhan D. S., "Implementation of fuzzy system using different voltages of OTA for JNK pathway leading to cell survival/ death", Network Biology, 5(2), 62-70 : 2015.

[7] Jain S, "Design and Simulation of Fuzzy System Using Two Stage CMOS Operational Amplifier", Journal of Active and Passive Electronic Devices, 2014, 9(4), 329-338.

[8] Jain S, "Implementation of Fuzzy System using Operational Transconductance Amplifier for ERK pathway of EGF/ Insulin leading to Cell Survival/ Death", J Pharm Biomed Sci, 2014, 4(8), 701-707.

[9] Berkan R.C., Trubatch S. L., Fuzzy System Design Principles, 1997.1st edition, Wiley-IEEE Press.

[10] John Yen,Reza Langari, Fuzzy Logic: intelligent Control and Information, 1998. United states ed edition, Prentice Hall.

[11] Rashid M.H., Introduction to PSICE using OrCAD for circuits and electronics, 2009. $3^{\text {rd }}$ edition, PHI. 